\title{
Journal of Organometallic Chemistry
}

journal homepage: www.elsevier.com

Calix[4]arene-assisted KOH-Catalyzed Synthesis of $O, O$-Dialkyl-Se-Aryl Phosphoroselenoates

Sihai Chen, Jinyang Chen, Xinhua Xu*, Yunhua He, Rongnan Yi, Renhua Qiu*

State Key Laboratory of Chemo/Biosensing and Chemometrics, College of Chemistry and Chemical Engineering Hunan University, Changsha, 410082, P.R.

China

Fax: (+ 86) 731-88821546; E-mail: xhx1581@hnu.edu.cn; renhuaqiu@hnu.edu.cn.

\section{ARTICLE INFO}

Article history:

Received

Received in revised form

Accepted

Available online

Keywords:

calix[4]arene 3

diaryl diselenide

$\boldsymbol{O}, \boldsymbol{O}$-dialkylphosphonate

$O, O$-dialkyl-Se-aryl phosphoroselenoate

KOH
ABSTRACT

A convenient and efficient method for the synthesis of $O, O$-dialkyl-Se-aryl phosphoroselenoates is described via a one pot reaction of diaryl diselenide and $O, O$ dialkylphosphonate catalyzed by $\mathrm{KOH}$ assisted by a co-catalyst of calix[4]arene 3 . The calix[4]arene 3 can be recycled for filve times with good yields of the desired products for the reaction. This protocol provide a simple and efficient method for the synthesis of $O, O$ dialkyl-Se-aryl phosphoroselenoates, avoiding the use of toxic reagents and expensive catalysts.

2016 Elsevier Ltd. All rights reserved.

\section{Introduction}

Due to the chemo-, regio- and stereoselective reactions and the useful biological activities, organoselenium compounds have become attractive synthetic targets in organic synthesis ${ }^{[1]}$. Among these organoselenium compounds, $O, O$-dialkyl-Se-aryl phosphoroselenoates are important intermediates in synthesis of natural products and complex compounds. ${ }^{[2]}$ As important functional compounds and attractive synthetic intermediates in organic synthesis, the synthesis and application of $O, O$-dialkylSe-aryl phosphoroselenoates have received considerable attentions $^{[3]}$. In 1996, Han et al. reported the reaction of phenyl lithium with $O, O$-diaryl phosphoryl chloride to prepare a series of $O, O$-dialkyl-Se-aryl phosphoroselenoates in good yields ${ }^{[4]}$. However, hard conditions were needed and toxic reagents were used in this procedure. Various methods using diaryl diselenides as starting materials were reported later ${ }^{[5]}$. However, these procedures are dangrous because of the use of explosive reagents (such as $\mathrm{AIBN}$ or $\mathrm{NaN}_{3} / \mathrm{PhI}(\mathrm{OAc})_{2}$ ) as radical initiators. A new method for the synthesis of $O, O$-dialkyl-Searyl phosphoroselenoates catalyzed by $\mathrm{CuI}$ was reported by $\mathrm{Gao}$ and co-workers ${ }^{[6]}$. However, at least one equivalent of organic base was used during this procedure. Therefore, a method that is efficient for the synthesis of these $O, O$-dialkyl-Se-aryl phosphoroselenoates is on demand. In our previous work ${ }^{[7]}$, we reported a convenient method for the synthesis of these useful compounds using diaryl diselenides and $O, O$ dialkylphosphonates as starting materials catalyzed by cesium hydroxide monohydrate. However, compared to other common alkali metal hydroxides (such as $\mathrm{NaOH}$ and $\mathrm{KOH}$ ), the high cost of the use of $\mathrm{CsOH}$ in this procedure hinder the development of this method in industrial application.

Compare to crown ethers ${ }^{[8]}$ and cyclodextrins ${ }^{[9]}$, calixarenes with the moderate cavity show stronger complexing ability to metal cations, which enhance the nucleophilicity of the nucleophilic reagents and promote the reaction efficiently. ${ }^{[10]}$ Calixarenes (such as $\mathbf{1}^{[11]}$ and $\mathbf{2}^{[12]}$ ) (Scheme 1) have been used as phase transform catalyst in organic synthesis for preparation of many useful products. ${ }^{[13]}$ Here, we report, in the first part, our results on the synthesis of $O, O$-dialkyl-Se-aryl phosphoroselenoates catalyzed by Calix[4]arene-assisted $\mathrm{KOH}$, which is $\mathrm{KOH}$ combined with an assisted co-catalyst of calix[4]arene 3. This procedure provides a convenient and economic method for the synthesis of $O, O$-dialkyl-Se-aryl phosphoroselenoates by using readily available reagents as the starting materials.
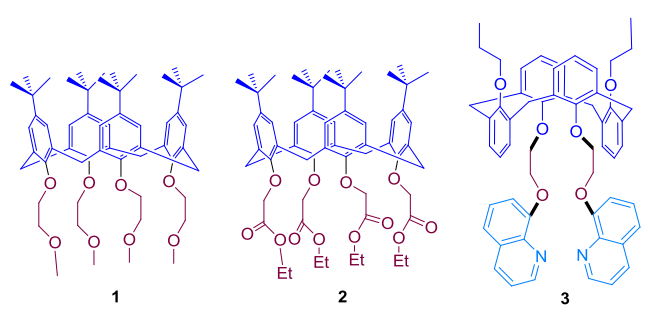

Scheme 1. Structures of calix[4] arene 1, 2 and $\mathbf{3}$

\section{Results and discussion}

\subsection{Synthesis of calix[4]arene $3^{[14]}$}

The sulfonation of 25,27-di(n-propyl)-26,28-dihydroxyl calix[4]arene (4) ${ }^{[15]}$ with sulphonate yields compound $\mathbf{5}$, which then converted to calix[4]arene $\mathbf{3}$ by treatment with 8hydroxyquinoline catalyzed by $\mathrm{K}_{2} \mathrm{CO}_{3}$ in the total yield of $54.2 \%$ (Scheme 2).

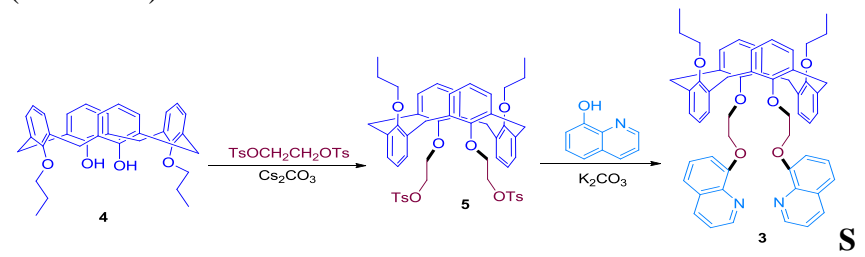

Scheme 2. The synthesis of calix[4]arene 3

2.2 Optimization of the reaction conditions for the selenation 
The reaction of $O, O$-dimethyl phosphonate (6a) with diphenyl diselenide (7a) was run at room temperature (rt) by using $10 \mathrm{~mol} \% \mathrm{TBAB}$ and $20 \mathrm{~mol} \% \mathrm{KOH}$ as co-catalyst, the desired product 8a was obtained in 19\% GC yield within $8 \mathrm{~h}$ (Table 1, entry 1). Then we examined a various of phase transfer catalysts to optimize the reaction conditions (Table 1, entries 1-6), and the results shown that calix[4]arene 3 was the best PTC for this reaction giving the desired product in the yield of $80 \%$ within $8 \mathrm{~h}$ (Table 1, entry6). To promote the reaction, a series of solvents (such as $\mathrm{CH}_{2} \mathrm{Cl}_{2}$, EtOH, DMF and THF) were explored (Table 1, entries 6-11), THF was turned out to be the best solvent, affording the desired product in $86 \%$ yield (Table 1 , entry 10). On the base of above results, we examined the amount of calix[4]arene 3 and $\mathrm{KOH}$ necessarily to promote the reaction efficiently (Table 1, entries 11-16), and found that 5.0 mol\% of calix[4]arene 3 and $20 \mathrm{~mol} \% \mathrm{KOH}$ was enough to afford the desired product in the yield of $90 \%$, when the reaction time was extended to $15 \mathrm{~h}$ (Table 1, entry 15) in open air, while the desired product was obtained in trace under the nitrogen atmosphere (Table 1, entries 17), implying the air is involved in the reaction. Thus, the optimized conditions are as follows: $5.0 \mathrm{~mol} \%$ of calix[4] arene $\mathbf{3}$ and $20 \mathrm{~mol} \%$ of $\mathrm{KOH}$ as co-catalyst, 1.0 equiv. of $7 \mathbf{a}$ and 2.2 equiv. of $\mathbf{6 a}$ in THF at rt for $15 \mathrm{~h}$ in air.

Table 1. Optimization of the reaction conditions for the selenation. ${ }^{\mathrm{a}}$

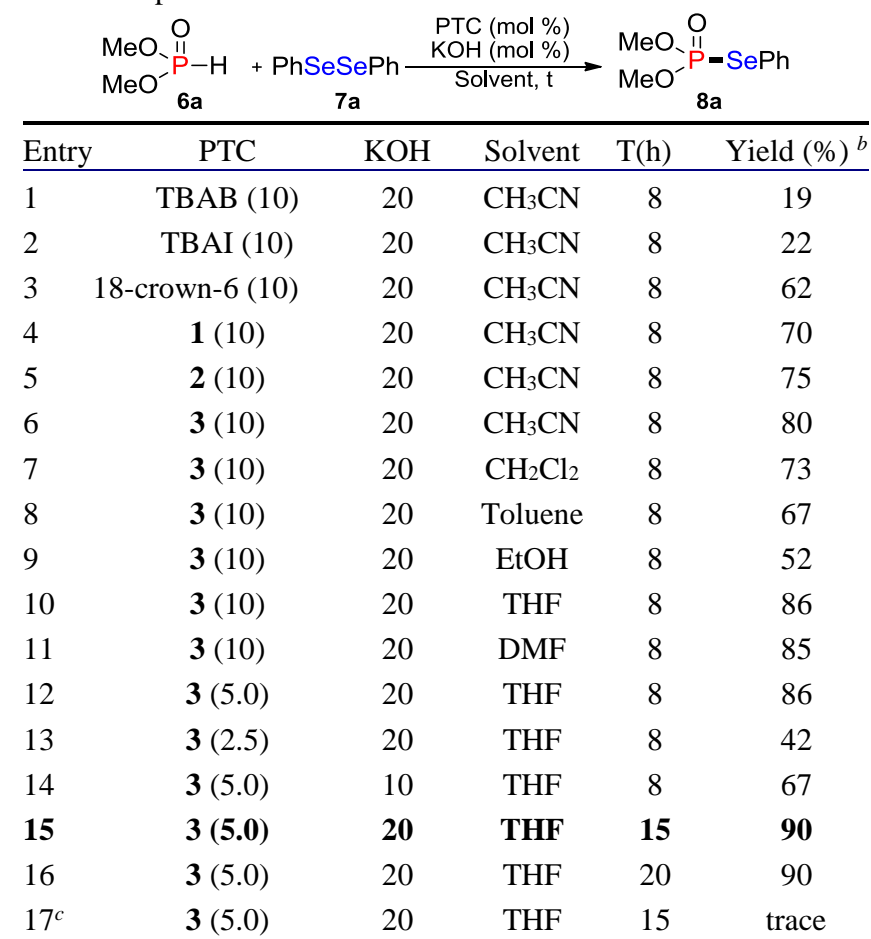

${ }^{a}$ Reaction conditions: 6a $(2.2 \mathrm{mmol}), 7 \mathbf{a}(1.0 \mathrm{mmol})$, solvent $(1.0 \mathrm{~mL}), \mathrm{rt}$, in air. ${ }^{b} \mathrm{GC}$ yields. ${ }^{c}$ The reaction was performed under a nitrogen amosphere.

\subsection{The scope of the selenation}

With an efficient protocol for the selenation in hand, we next investigated its scope with different $O, O$-dialkylphosphonate (6) and diaryl diselenide (7). As shown in Table 2, we can find that the reaction of a variety of $O, O$-dialkylphosphonate $(\mathbf{6})$ and diaryl diselenide (7) could undergo efficiently to desired products in good to excellent yields. A closer inspection of the results revealed that the functional groups $(\mathrm{Me}, \mathrm{Et}, i$-Pr and $n$ $\mathrm{Bu}$ ) presenting in $O, O$-dialkylphosphonates effect the reaction slightly, and all the desired products were obtained in excellent yields (85\%-92\%, 8a-8d). In addition, the reaction is not sensitive to the nature of functional groups $(\mathrm{Me}, \mathrm{Cl})$ presenting in the benzene rings of the diaryl diselenides, and all the reactions afford the desired products in good yields $(83 \%-89 \%$, $8 \mathrm{e}-8 \mathbf{j})$.

Table 2. The selenation of $O, O$-dialkylphosphonate (4) catalyzed by calix[4]arene $3(5 \mathrm{~mol} \%) / \mathrm{KOH}(20 \mathrm{~mol} \%)$ in THF. ${ }^{\mathrm{a}}$

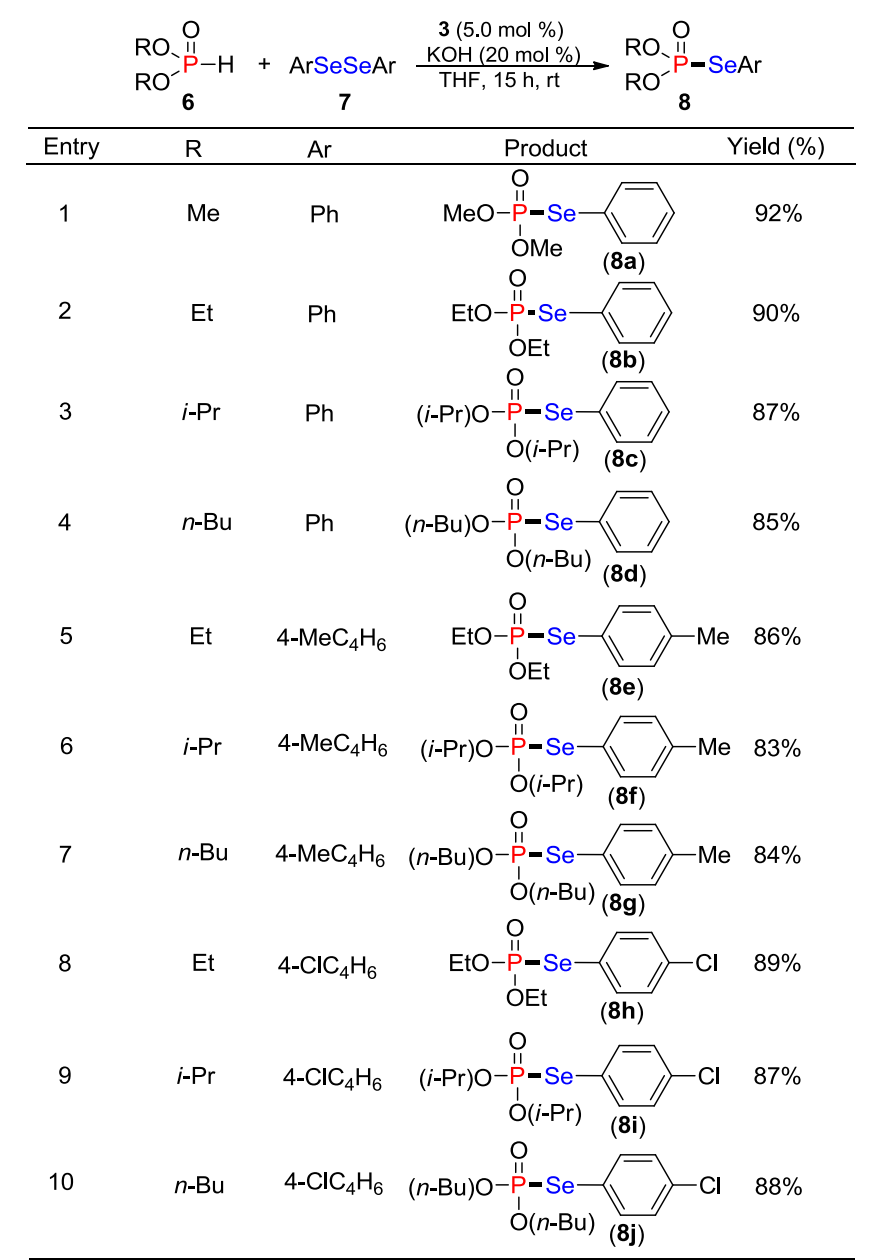

${ }^{a}$ Reaction conditions: $6(2.2 \mathrm{mmol}), \mathbf{7}(1.0 \mathrm{mmol}), \mathrm{KOH}(20 \mathrm{~mol} \%), 3(5.0$ $\mathrm{mol} \%)$, THF $(2 \mathrm{~mL}), 25^{\circ} \mathrm{C}$, in air for $15 \mathrm{~h} .{ }^{b}$ Isolated yields.

\subsection{Cycles of calix[4]arene 3}

To test the reusability and reproducibility, the calix[4] arene $\mathbf{3}$ is subjected to cycles of selenation of $O, O$-diethyl phosphonate (6a) as the model reaction. After completion of the reaction, calix[4]arene 3 were obtained by flash chromatography. After separating, the calix[4]arene $\mathbf{3}$ is used for next cycle of the reaction. The decline in product yield is minimal in a run of 5 cycles $(90 \%$ to $85 \%)$, indicating that the calix[4]arene $\mathbf{3}$ is stable and suitable for reuse (Table 3).

Table 3. Yields of selenation of $O, O$-dialkylphosphonate by recover catalyst ${ }^{\mathrm{a}}$

\begin{tabular}{cc}
\hline Cycle & Yield $(\%)^{\mathrm{b}}$ \\
\hline 1 & 90 \\
2 & 90 \\
3 & 88 \\
4 & 86 \\
5 & 85 \\
\hline
\end{tabular}

${ }^{a}$ Reaction conditions: 6a $(2.2 \mathrm{mmol}), 7 \mathbf{a}(1.0 \mathrm{mmol})$, solvent $(1.0 \mathrm{~mL}), \mathrm{rt}$, in air. ${ }^{b}$ Isolated yield.

\subsection{The possible mechanism}

To shed light on the mechanism of the reaction, we compared the ${ }^{1} \mathrm{H}$ NMR spectrum of the calix[4]arene $\mathbf{3}$ and its complex 
with potassium picrate (see spectrums in supplementary material). The area under a picrate anion peak at $\delta 8.44$ was found, implying that the calix[4]arene 3-potassium ion complex was formed in chloroform. The changes in chemical shift and peak collapsing also indicated the strong complexation between calix[4]arene 3 with $\mathrm{K}^{+}{ }^{[16]}$ which enhances the nucleophilicity of the $\mathrm{KOH}$, and consequently promote the reaction efficiently. The desired product was obtained in poor yield when the reaction was performed under the optimized conditions except for a nitrogen atmosphere, which indicated that oxygen is needed during the selenation (Table 1, entries 16-17).

Base on the results described above and previous reports ${ }^{[17]}$ a mechanism for this reaction is proposed in Scheme 2. The complexation of calix[4]arene $\mathbf{3}$ with $\mathrm{K}^{+}$enhances the nucleophilicity of $\mathrm{KOH}$ and reacted with $O, O$ dialkylphosphonate to give intermediate $\boldsymbol{a}$, which reacted with diaryl diselenides to yield $O, O$-dialkyl-Se-aryl phosphoroselenoates and organoselenium anion $\boldsymbol{b}$. The organoselenium anion $\boldsymbol{b}$ can be converted to diaryl diselenides after the oxidation in air ${ }^{[18]}$.
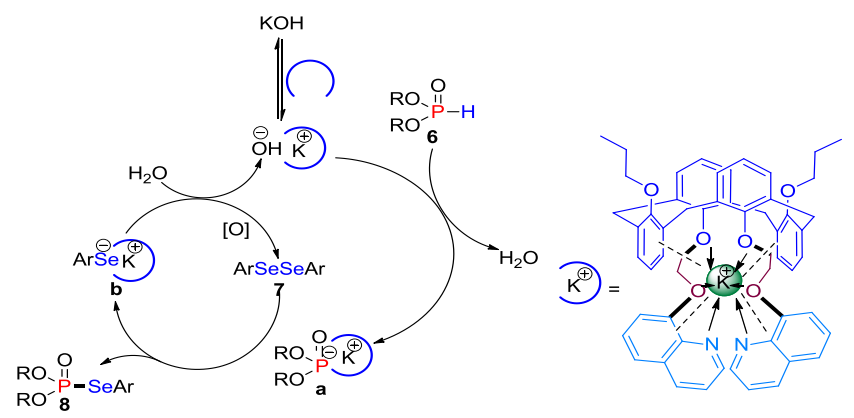

Scheme 3. The possible mechanism of the selenation

\section{Conclusions}

In summary, we have developed a convenient and low-cost method for the synthesis of $O, O$-dialkyl-Se-aryl phosphoroselenoates catalyzed by calix[4]arene-assisted $\mathrm{KOH}$ by using easily available and cheap reagents as starting materials. The other advantages of this procedure include mild conditions, high yields and wide functional group tolerance as well as recyclable calix[4]arene co-catalyst.

\section{Experimental}

\subsection{General}

Reactions were monitored by thin layer chromatography (TLC) using gel 60 F254 pre-coated plates. Visualization was accomplished with UV lamp or $\mathrm{I}_{2}$ stain. Silica gel 300-400 mesh size was used for column chromatography using the combination of ethyl acetate and petroleum ether as an eluent. ${ }^{1} \mathrm{H}$ NMR and ${ }^{13} \mathrm{C}$ NMR spectra were recorded on $400 \mathrm{MHz}$ and NMR plus spectrometer using residue solvent peaks as internal standards respectively. Mass spectra (MS) were obtained using EI mass spectrometer. High-resolution mass spectra (HRMS) were measured on an electron ionization (EI) mass spectrometer.

\subsection{Synthesis of calix[4]arene 3}

To a solution of 25,27-di(n-propyl)-26,28-dihydroxyl calix[4]arene (4) ${ }^{[15]}(1.0 \mathrm{~g}, 2.0 \mathrm{mmol})$ in $50 \mathrm{~mL}$ of dry $\mathrm{CH}_{3} \mathrm{CN}$ was added 1,2-di(4-methyl acyloxy) ethane (1.55 g, $4.2 \mathrm{mmol})$ and $\mathrm{Cs}_{2} \mathrm{CO}_{3}(3.26 \mathrm{~g}, 10 \mathrm{mmol})$. The mixture solution was refluxed under a nitrogen atmosphere for 24 hours. Then the reaction solution was treated with $\mathrm{H}_{2} \mathrm{O}(30 \mathrm{~mL})$ and extracted with $\mathrm{CH}_{2} \mathrm{Cl}_{2}$. The combined organic phase was dried over anhydrous $\mathrm{Na}_{2} \mathrm{SO}_{4}$ and concentrated under vacuum. The desired products compound $\mathbf{5}$ was obtained by flash chromatography using ethyl acetate/hexane as an eluent. Then, 8-hydroxyquinoline $(0.69 \mathrm{~g}, 4.2 \mathrm{mmol})$ was added to the solution of compound 5 and $\mathrm{K}_{2} \mathrm{CO}_{3}(1.38 \mathrm{~g}, 10 \mathrm{mmol})$. The reaction mixture was refluxed for 24 hours under a nitrogen atmosphere. Then the reaction solution was treated with $\mathrm{H}_{2} \mathrm{O}$ (30 $\mathrm{mL}$ ) and extracted with $\mathrm{CH}_{2} \mathrm{Cl}_{2}$. The combined organic phase was dried over anhydrous $\mathrm{Na}_{2} \mathrm{SO}_{4}$ and concentrated under vacuum. The calix[4]arene 3 was obtained in the total yield of $54.2 \%$ by flash chromatography using ethyl acetate/hexane as an eluent. ${ }^{1} \mathrm{H}$ $\operatorname{NMR}\left(400 \mathrm{MHz}, \mathrm{CDCl}_{3}\right) \delta 0.84(\mathrm{t}, J=8.2 \mathrm{~Hz}, 3 \mathrm{H}), 1.51-1.55(\mathrm{~m}$, $2 \mathrm{H}), 3.48(\mathrm{t}, J=8.2 \mathrm{~Hz}, 2 \mathrm{H}), 3.68-3.80(\mathrm{~m}, 4 \mathrm{H}), 4.22(\mathrm{t}, J=6.4 \mathrm{~Hz}$, $2 \mathrm{H}), 4.28(\mathrm{t}, J=4.3 \mathrm{~Hz}, 2 \mathrm{H}), 6.46(\mathrm{t}, J=6.3 \mathrm{~Hz}, 1 \mathrm{H}), 6.76(\mathrm{t}, J=$ $8.4 \mathrm{~Hz}, 1 \mathrm{H}), 7.04(\mathrm{~d}, J=8.5 \mathrm{~Hz}, 2 \mathrm{H}), 7.07(\mathrm{t}, J=4.2 \mathrm{~Hz}, 1 \mathrm{H}), 7.10$ $(\mathrm{d}, J=8.2 \mathrm{~Hz}, 2 \mathrm{H}), 7.38(\mathrm{~d}, J=4.4 \mathrm{~Hz}, 2 \mathrm{H}), 7.42-7.45(\mathrm{~m}, 1 \mathrm{H})$, $8.14\left(\mathrm{dd}, J_{1}=1.3 \mathrm{~Hz}, J_{2}=1.4 \mathrm{~Hz}, 1 \mathrm{H}\right), 8.96\left(\mathrm{dd}, J_{1}=1.2 \mathrm{~Hz}, J_{2}=\right.$ $1.0 \mathrm{~Hz}, 1 \mathrm{H}) ;{ }^{13} \mathrm{C} \mathrm{NMR}\left(100 \mathrm{MHz}, \mathrm{CDCl}_{3}\right) \delta 8.61,12.50,21.07$, $21.13,21.55, \quad 27.70,27.96, \quad 28.08, \quad 35.48,66.23,67.73$, $71.26,108.24,118.41,120.11,120.66,125.42,127.95,132.13$, 134.50, 147.70, 154.28, 155.06; Ms (m/z): 851.4.

\subsection{Typical procedure for the synthesis of O,O-dialkyl-Se-aryl phosphoroselenoate}

In a round bottomed flask, calix[4] arene $3(0.05 \mathrm{mmol})$ and $\mathrm{KOH}$ $(0.2 \mathrm{mmol})$ was added to a solution of $O, O$-dialkylphosphonates $(2.2 \mathrm{mmol})$ and diaryl diselenides $(1.0 \mathrm{mmol})$ in THF $(2.0 \mathrm{~mL})$. The reaction mixture was stirred at $\mathrm{rt}$ in air for 15 hours. After finish, the reaction solution was dried over anhydrous $\mathrm{Na}_{2} \mathrm{SO}_{4}$ and concentrated under vacuum. The desired products 8 and calix[4]arene 3 were obtained by flash chromatography using ethyl acetate/hexane as an eluent.

O,O-dimethyl-Se-phenyl phosphoroselenoate $(8 a): \quad{ }^{1} \mathrm{H} \quad \mathrm{NMR}$ $\left(\mathrm{CDCl}_{3}, 400 \mathrm{MHz}\right): 7.64(\mathrm{~d}, J=8.0 \mathrm{~Hz}, 2 \mathrm{H}), 7.37-7.30(\mathrm{~m}, 3 \mathrm{H})$, 3.81 (s, 3H), 3.78 (s, 3H) ppm; ${ }^{13} \mathrm{C} \mathrm{NMR}\left(\mathrm{CDCl}_{3}, 100 \mathrm{MHz}\right): 134.6$ $\left(\mathrm{d}, J_{\mathrm{C}-\mathrm{P}}=4.8 \mathrm{~Hz}, 1 \mathrm{C}\right), 129.5\left(\mathrm{~d}, J_{\mathrm{C}-\mathrm{P}}=2.1 \mathrm{~Hz}, 2 \mathrm{C}\right), 129.2\left(\mathrm{~d}, J_{\mathrm{C}-\mathrm{P}}=\right.$ $2.6 \mathrm{~Hz}, 2 \mathrm{C}), 126.0\left(\mathrm{~d}, J_{\mathrm{C}-\mathrm{P}}=8.4 \mathrm{~Hz}, 1 \mathrm{C}\right), 54.0\left(\mathrm{~d}, J_{\mathrm{C}-\mathrm{P}}=5.7 \mathrm{~Hz}\right.$, 2C) ppm; ${ }^{31} \mathrm{P}$ NMR (162 MHz, $\left.\mathrm{CDCl}_{3}\right): 21.97 \mathrm{ppm}$; $\mathrm{MS}(\mathrm{EI}): \mathrm{m} / \mathrm{z}=$ 266.0. HRMS calc. for $\mathrm{C}_{8} \mathrm{H}_{11} \mathrm{O}_{3} \mathrm{PSe} 265.9606$, found 265.9612 .

O,O-dimethyl-Se-phenyl phosphoroselenoate $(8 \boldsymbol{b}):{ }^{1} \mathrm{H} \quad \mathrm{NMR}$ $\left(\mathrm{CDCl}_{3}, 400 \mathrm{MHz}\right)$ : 7.65-7.63 (m, 2H), 7.385-7.29 (m, 3H), 4.24$4.12(\mathrm{~m}, 4 \mathrm{H}), 1.30(\mathrm{t}, J=7.0 \mathrm{~Hz}, 6 \mathrm{H}) \mathrm{ppm} ;{ }^{13} \mathrm{C} \mathrm{NMR}\left(\mathrm{CDCl}_{3}, 100\right.$ $\mathrm{MHz}): 134.5\left(\mathrm{~d}, J_{\mathrm{C}-\mathrm{P}}=4.6 \mathrm{~Hz}, 1 \mathrm{C}\right), 129.5\left(\mathrm{~d}, J_{\mathrm{C}-\mathrm{P}}=2.0 \mathrm{~Hz}, 2 \mathrm{C}\right)$, $128.8\left(\mathrm{~d}, J_{\mathrm{C}-\mathrm{P}}=2.5 \mathrm{~Hz}, 2 \mathrm{C}\right), 123.8\left(\mathrm{~d}, J_{\mathrm{C}-\mathrm{P}}=8.4 \mathrm{~Hz}, 1 \mathrm{C}\right), 63.9(\mathrm{~d}$, $\left.J_{\mathrm{C}-\mathrm{P}}=5.8 \mathrm{~Hz}, 2 \mathrm{C}\right), 15.9\left(\mathrm{~d}, J_{\mathrm{C}-\mathrm{P}}=7.4 \mathrm{~Hz}, 2 \mathrm{C}\right) \mathrm{ppm} ;{ }^{31} \mathrm{P}$ NMR $(162$ $\left.\mathrm{MHz}, \mathrm{CDCl}_{3}\right): 17.97 \mathrm{ppm}$; MS (EI): $\mathrm{m} / \mathrm{z}=294.0$. HRMS calc. for $\mathrm{C}_{10} \mathrm{H}_{15} \mathrm{O}_{3} \mathrm{PSe} 293.9919$, found 193.9930.

O,O-di(i-propyl)-Se-phenyl phosphoroselenoate $(8 \mathrm{c}):{ }^{1} \mathrm{H}$ NMR $\left(\mathrm{CDCl}_{3}, 400 \mathrm{MHz}\right): 7.66(\mathrm{~d}, J=7.6 \mathrm{~Hz}, 2 \mathrm{H}), 7.35-7.28(\mathrm{~m}, 3 \mathrm{H})$, 4.84-4.72 (m, 2H), $1.33(\mathrm{~d}, J=6.4 \mathrm{~Hz}, 6 \mathrm{H}), 1.25(\mathrm{~d}, J=6.4 \mathrm{~Hz}$, $6 \mathrm{H}) \mathrm{ppm} ;{ }^{13} \mathrm{C} \mathrm{NMR}\left(\mathrm{CDCl}_{3}, 100 \mathrm{MHz}\right): 135.1\left(\mathrm{~d}, J_{\mathrm{C}-\mathrm{P}}=4.9 \mathrm{~Hz}\right.$, $1 \mathrm{C}), 129.3\left(\mathrm{~d}, J_{\mathrm{C}-\mathrm{P}}=1.8 \mathrm{~Hz}, 2 \mathrm{C}\right), 128.5\left(\mathrm{~d}, J_{\mathrm{C}-\mathrm{P}}=2.3 \mathrm{~Hz}, 2 \mathrm{C}\right)$, $124.6\left(\mathrm{~d}, J_{\mathrm{C}-\mathrm{P}}=8.2 \mathrm{~Hz}, 1 \mathrm{C}\right), 73.1\left(\mathrm{~d}, J_{\mathrm{C}-\mathrm{P}}=6.6 \mathrm{~Hz}, 2 \mathrm{C}\right), 23.8(\mathrm{~d}$, $\left.J_{\mathrm{C}-\mathrm{P}}=3.7 \mathrm{~Hz}, 2 \mathrm{C}\right), 23.5\left(\mathrm{~d}, J_{\mathrm{C}-\mathrm{P}}=6.0 \mathrm{~Hz}, 2 \mathrm{C}\right) \mathrm{ppm} ;{ }^{31} \mathrm{P}$ NMR $(162$ $\mathrm{MHz}, \mathrm{CDCl}_{3}$ ): $14.69 \mathrm{ppm}$; MS (EI): $\mathrm{m} / \mathrm{z}=322.1$. HRMS calc. for $\mathrm{C}_{12} \mathrm{H}_{19} \mathrm{O}_{3} \mathrm{PSe} 322.0232$, found 322.0228 .

O,O-di(n-butyl)-Se-phenyl phosphoroselenoate (8d): ${ }^{1} \mathrm{H}$ NMR $\left(\mathrm{CDCl}_{3}, 400 \mathrm{MHz}\right)$ : 7.65-7.63 (m, 2H), 7.37-7.27 (m, 3H), 4.17$4.03(\mathrm{~m}, 4 \mathrm{H}), 1.66-1.58(\mathrm{~m}, 4 \mathrm{H}), 1.39-1.29(\mathrm{~m}, 4 \mathrm{H}), 0.89(\mathrm{t}, J=7.4$ $\mathrm{Hz}, 6 \mathrm{H}) ;{ }^{13} \mathrm{C}$ NMR $\left(\mathrm{CDCl}_{3}, 100 \mathrm{MHz}\right): 135.5\left(\mathrm{~d}, J_{\mathrm{C}-\mathrm{P}}=4.7 \mathrm{~Hz}\right.$, $1 \mathrm{C}), 129.4\left(\mathrm{~d}, J_{\mathrm{C}-\mathrm{P}}=2.0 \mathrm{~Hz}, 2 \mathrm{C}\right), 128.7\left(\mathrm{~d}, J_{\mathrm{C}-\mathrm{P}}=2.5 \mathrm{~Hz}, 2 \mathrm{C}\right)$, $123.8\left(\mathrm{~d}, J_{\mathrm{C}-\mathrm{P}}=8.3 \mathrm{~Hz}, 1 \mathrm{C}\right), 67.6\left(\mathrm{~d}, J_{\mathrm{C}-\mathrm{P}}=6.3 \mathrm{~Hz}, 2 \mathrm{C}\right), 32.0(\mathrm{~d}$, $\left.J_{\mathrm{C}-\mathrm{P}}=7.2 \mathrm{~Hz}, 2 \mathrm{C}\right), 18.6(\mathrm{~s}, 2 \mathrm{C}), 13.5(\mathrm{~s}, 2 \mathrm{C}) \mathrm{ppm} ;{ }^{31} \mathrm{P}$ NMR $(162$ $\mathrm{MHz}, \mathrm{CDCl}_{3}$ ): $18.07 \mathrm{ppm}$; MS (EI): $\mathrm{m} / \mathrm{z}=350.0$. HRMS calc. for $\mathrm{C}_{14} \mathrm{H}_{23} \mathrm{O}_{3} \mathrm{PSe} 350.0545$, found 350.0541 .

O,O-dimethyl-Se-(4-methylphenyl) phosphoroselenoate $(8 \boldsymbol{e}):{ }^{1} \mathrm{H}$ NMR $\left(\mathrm{CDCl}_{3}, 400 \mathrm{MHz}\right): 7.44(\mathrm{~d}, J=6.8 \mathrm{~Hz}, 2 \mathrm{H}), 7.05(\mathrm{~d}, J=7.6$ $\mathrm{Hz}, 2 \mathrm{H}), 4.18-4.03(\mathrm{~m}, 4 \mathrm{H}), 2.27(\mathrm{~s}, 3 \mathrm{H}), 1.24(\mathrm{t}, J=7.0 \mathrm{~Hz}, 6 \mathrm{H})$ 
ppm; ${ }^{13} \mathrm{C} \mathrm{NMR}\left(\mathrm{CDCl}_{3}, 100 \mathrm{MHz}\right): 138.0\left(\mathrm{~d}, J_{\mathrm{C}-\mathrm{P}}=3.5 \mathrm{~Hz}, 1 \mathrm{C}\right)$, $134.6\left(\mathrm{~d}, J_{\mathrm{C}-\mathrm{P}}=4.4 \mathrm{~Hz}, 2 \mathrm{C}\right), 129.3\left(\mathrm{~d}, J_{\mathrm{C}-\mathrm{P}}=2.1 \mathrm{~Hz}, 2 \mathrm{C}\right), 119.0(\mathrm{~d}$, $\left.J_{\mathrm{C}-\mathrm{P}}=7.5 \mathrm{~Hz}, 1 \mathrm{C}\right), 62.7\left(\mathrm{~d}, J_{\mathrm{C}-\mathrm{P}}=5.8 \mathrm{~Hz}, 2 \mathrm{C}\right), 14.9\left(\mathrm{~d}, J_{\mathrm{C}-\mathrm{P}}=7.4\right.$ $\mathrm{Hz}, 2 \mathrm{C}$ ) ppm; ${ }^{31} \mathrm{P}$ NMR (162 MHz, $\left.\mathrm{CDCl}_{3}\right): 18.37 \mathrm{ppm}$; MS (EI): $\mathrm{m} / \mathrm{z}=308.0$. HRMS calc. for $\mathrm{C}_{11} \mathrm{H}_{17} \mathrm{O}_{3} \mathrm{PSe}$ 308.0075, found 308.0084 .

O,O-di(i-propyl)-Se-(4-methylphenyl) phosphoroselenoate $(8 f):{ }^{1} \mathrm{H}$ NMR $\left(\mathrm{CDCl}_{3}, 400 \mathrm{MHz}\right)$ : 7.55-7.52 (m, 2H), 7.11 (d, $J=7.6 \mathrm{~Hz}$, $2 \mathrm{H}), 4.82-4.73(\mathrm{~m}, 2 \mathrm{H}), 2.33(\mathrm{~s}, 3 \mathrm{H}), 1.34(\mathrm{~d}, J=6.4 \mathrm{~Hz}, 6 \mathrm{H}), 1.26$ $(\mathrm{d}, J=6.4 \mathrm{~Hz}, 6 \mathrm{H}) \mathrm{ppm} ;{ }^{13} \mathrm{C} \mathrm{NMR}\left(\mathrm{CDCl}_{3}, 100 \mathrm{MHz}\right): 138.6(\mathrm{~d}$, $\left.J_{\mathrm{C}-\mathrm{P}}=2.7 \mathrm{~Hz}, 1 \mathrm{C}\right), 135.3\left(\mathrm{~d}, J_{\mathrm{C}-\mathrm{P}}=4.7 \mathrm{~Hz}, 2 \mathrm{C}\right), 130.1\left(\mathrm{~d}, J_{\mathrm{C}-\mathrm{P}}=1.9\right.$ $\mathrm{Hz}, 2 \mathrm{C}), 120.7\left(\mathrm{~d}, J_{\mathrm{C}-\mathrm{P}}=8.4 \mathrm{~Hz}, 1 \mathrm{C}\right), 73.0\left(\mathrm{~d}, J_{\mathrm{C}-\mathrm{P}}=6.6 \mathrm{~Hz}, 2 \mathrm{C}\right)$, $23.9\left(\mathrm{~d}, J_{\mathrm{C}-\mathrm{P}}=3.7 \mathrm{~Hz}, 2 \mathrm{C}\right), 23.5\left(\mathrm{~d}, J_{\mathrm{C}-\mathrm{P}}=5.9 \mathrm{~Hz}, 2 \mathrm{C}\right), 21.1(\mathrm{~s}, 1 \mathrm{C})$ ppm; ${ }^{31} \mathrm{P}$ NMR (162 MHz, $\left.\mathrm{CDCl}_{3}\right): 15.06 \mathrm{ppm}$; MS (EI): m/z =336.0. HRMS calc. for $\mathrm{C}_{13} \mathrm{H}_{21} \mathrm{O}_{3} \mathrm{PSe} 336.0388$, found 336.0379.

O,O-di(n-butyl)-Se-(4-methylphenyl) phosphoroselenoate $(8 g):{ }^{1} \mathrm{H}$ $\operatorname{NMR}\left(\mathrm{CDCl}_{3}, 400 \mathrm{MHz}\right): 7.52(\mathrm{~d}, J=6.8 \mathrm{~Hz}, 2 \mathrm{H}), 7.114(\mathrm{~d}, J=$ $8.0 \mathrm{~Hz}, 2 \mathrm{H}), 4.16-4.03$ (m, 4H), 2.33 (s, 3H), 1.66-1.59 (m, 4H), $1.39-1.30(\mathrm{~m}, 4 \mathrm{H}), 0.90(\mathrm{t}, J=7.4 \mathrm{~Hz}, 6 \mathrm{H}) \mathrm{ppm} ;{ }^{13} \mathrm{C} \mathrm{NMR}\left(\mathrm{CDCl}_{3}\right.$, $100 \mathrm{MHz}): 138.9\left(\mathrm{~d}, J_{\mathrm{C}-\mathrm{P}}=2.9 \mathrm{~Hz}, 1 \mathrm{C}\right), 135.6\left(\mathrm{~d}, J_{\mathrm{C}-\mathrm{P}}=4.4 \mathrm{~Hz}\right.$, 2C), $130.2\left(\mathrm{~d}, J_{\mathrm{C}-\mathrm{P}}=2.2 \mathrm{~Hz}, 2 \mathrm{C}\right), 120.0\left(\mathrm{~d}, J_{\mathrm{C}-\mathrm{P}}=8.5 \mathrm{~Hz}, 1 \mathrm{C}\right), 67.5$ $\left(\mathrm{d}, J_{\mathrm{C}-\mathrm{P}}=6.3 \mathrm{~Hz}, 2 \mathrm{C}\right), 32.0\left(\mathrm{~d}, J_{\mathrm{C}-\mathrm{P}}=7.3 \mathrm{~Hz}, 2 \mathrm{C}\right), 21.2(\mathrm{~s}, 1 \mathrm{C})$, 18.7 (s, 2C), 13.5 (s, 2C) ppm; ${ }^{31} \mathrm{P}$ NMR (162 $\left.\mathrm{MHz}, \mathrm{CDCl}_{3}\right)$ : 18.41ppm; MS (EI): $\mathrm{m} / \mathrm{z}=364.1$. HRMS calc. for $\mathrm{C}_{15} \mathrm{H}_{25} \mathrm{O}_{3} \mathrm{PSe}$ 364.0701, found 364.0695 .

O,O-diethyl-Se-(4-chlorophenyl) phosphoroselenoate $(8 \boldsymbol{h}):{ }^{1} \mathrm{H}$ $\operatorname{NMR}\left(400 \mathrm{MHz}, \mathrm{CDCl}_{3}\right) \delta 7.58(\mathrm{~d}, J=7.2 \mathrm{~Hz}, 2 \mathrm{H}), 7.29(\mathrm{~d}, J=$ $8.0 \mathrm{~Hz}, 2 \mathrm{H}), 4.22-4.15(\mathrm{~m}, 4 \mathrm{H}), 1.32(\mathrm{t}, J=7.2 \mathrm{~Hz}, 6 \mathrm{H}) \mathrm{ppm} ;{ }^{13} \mathrm{C}$ NMR $\left(100 \mathrm{MHz}, \mathrm{CDCl}_{3}\right) \delta 136.78\left(\mathrm{~d}, J_{\mathrm{C}-\mathrm{P}}=4.7 \mathrm{~Hz}, 1 \mathrm{C}\right), 135.33$ $\left(\mathrm{d}, J_{\mathrm{C}-\mathrm{P}}=3.0 \mathrm{~Hz}, 1 \mathrm{C}\right), 129.67\left(\mathrm{~d}, J_{\mathrm{C}-\mathrm{P}}=2.1 \mathrm{~Hz}, 1 \mathrm{C}\right), 121.95\left(\mathrm{~d}, J_{\mathrm{C}-\mathrm{P}}\right.$ $=8.6 \mathrm{~Hz}, 1 \mathrm{C}), 64.01\left(\mathrm{~d}, J_{\mathrm{C}-\mathrm{P}}=6.0 \mathrm{~Hz}, 1 \mathrm{C}\right), 15.94\left(\mathrm{~d}, J_{\mathrm{C}-\mathrm{P}}=7.2 \mathrm{~Hz}\right.$, 1C) ppm; ${ }^{31} \mathrm{P}$ NMR (162 MHz, $\left.\mathrm{CDCl}_{3}\right): 19.52 \mathrm{ppm}$; $\mathrm{MS}(\mathrm{EI}): \mathrm{m} / \mathrm{z}=$ 327.9. HRMS calc. for $\mathrm{C}_{10} \mathrm{H}_{14} \mathrm{ClO}_{3} \mathrm{PSe} 327.9342$, found 327.9338 .

O,O-di(i-propyl)-Se-(4-chlorophenyl) phosphoroselenoate $(8 \mathrm{8i}):{ }^{1} \mathrm{H}$ $\operatorname{NMR}\left(400 \mathrm{MHz}, \mathrm{CDCl}_{3}\right) \delta 7.53(\mathrm{~d}, J=7.59 \mathrm{~Hz}, 2 \mathrm{H}), 7.20(\mathrm{~d}, J=$ $8.0 \mathrm{~Hz}, 2 \mathrm{H}), 4.76-4.650(\mathrm{~m}, 2 \mathrm{H}), 1.27(\mathrm{~d}, J=6.0 \mathrm{~Hz}, 6 \mathrm{H}), 1.20(\mathrm{~d}$, $J=6.4 \mathrm{~Hz}, 6 \mathrm{H}) \mathrm{ppm} ;{ }^{13} \mathrm{C} \mathrm{NMR}\left(100 \mathrm{MHz}, \mathrm{CDCl}_{3}\right) \delta 136.45\left(\mathrm{~d}, J_{\mathrm{C}}\right.$ $\mathrm{P}=4.9 \mathrm{~Hz}, 1 \mathrm{C}), 135.02\left(\mathrm{~d}, J_{\mathrm{C}-\mathrm{P}}=3.2 \mathrm{~Hz}, 1 \mathrm{C}\right), 129.54\left(\mathrm{~d}, J_{\mathrm{C}-\mathrm{P}}=1.8\right.$ $\mathrm{Hz}, 1 \mathrm{C}), 122.77\left(\mathrm{~d}, J_{\mathrm{C}-\mathrm{P}}=8.4 \mathrm{~Hz}, 1 \mathrm{C}\right), 73.38\left(\mathrm{~d}, J_{\mathrm{C}-\mathrm{P}}=6.8 \mathrm{~Hz}, 1 \mathrm{C}\right)$, $23.86\left(\mathrm{~d}, J_{\mathrm{C}-\mathrm{P}}=3.9 \mathrm{~Hz}, 1 \mathrm{C}\right), 23.52\left(\mathrm{~d}, J_{\mathrm{C}-\mathrm{P}}=5.8 \mathrm{~Hz}, 1 \mathrm{C}\right) \mathrm{ppm} ;{ }^{31} \mathrm{P}$ NMR (162 MHz, $\left.\mathrm{CDCl}_{3}\right): 20.12 \mathrm{ppm}$; MS (EI): $\mathrm{m} / \mathrm{z}=356.4$. HRMS calc. for $\mathrm{C}_{12} \mathrm{H}_{18} \mathrm{ClO}_{3} \mathrm{PSe} 356.4138$, found 356.4142 .

O,O-di(n-butyl)-Se-(4-chlorophenyl) phosphoroselenoate (8j): ${ }^{1} \mathrm{H}$ NMR (400 MHz, DMSO- $\left.d_{6}\right) \delta$ 7.65-7.63 (m, 2H), $7.45(\mathrm{~d}, J=8.4$ $\mathrm{Hz}, 2 \mathrm{H}), 4.09-4.01(\mathrm{~m}, 4 \mathrm{H}), 1.59-1.52(\mathrm{~m}, 4 \mathrm{H}), 1.32-1.23(\mathrm{~m}, 4 \mathrm{H})$; ${ }^{13} \mathrm{C}$ NMR (100 MHz, DMSO- $\left.d_{6}\right) \delta 137.42(\mathrm{~d}, J=4.59 \mathrm{~Hz}), 134.64$ $(\mathrm{d}, J=2.89 \mathrm{~Hz}), 130.08(\mathrm{~d}, J=1.8 \mathrm{~Hz}), 122.54(\mathrm{~d}, J=8.29 \mathrm{~Hz})$, $67.79(\mathrm{~d}, J=6.49 \mathrm{~Hz}), 31.89(\mathrm{~d}, J=6.89 \mathrm{~Hz}), 18.61,13.77 ;{ }^{31} \mathrm{P}$ NMR (162 MHz, DMSO- $d_{6}$ ): 20.23. MS (EI): $\mathrm{m} / \mathrm{z}=384.0$. HRMS calc. for $\mathrm{C}_{14} \mathrm{H}_{22} \mathrm{ClO}_{3} \mathrm{PSe} 384.0842$, found 384.0839 .

\section{Acknowledgements}

The project was supported by the National Science Foundation of China (Nos. 21273068 and 21373003) for financial support.

\section{Appendix A. Supplementary material}

All compounds are known from the literature, the copies of ${ }^{1} \mathrm{H}$ NMR and ${ }^{13} \mathrm{C}$ NMR spectra can be found via the "Supplementary Content" section of this article's webpage.

\section{References and notes}

[1] (a) G. Zeni, M. P. Stracke, C. W. Nogueira, A. L. Braga, P. H. Menezes, H. A. Stefani, Org. Lett. 6 (2004) 1135; (b) S. Liang, J. Chen, D. T. Pierce, J. X. Zhao, ACS Appl. Mater. Interfaces 5 (2013) 5165; (c) A. L. Eastwood, A. P. Blum, N. M. Zacharias, D. A. Dougherty, J. Org. Chem. 74 (2009) 9241; (d) C. W. Nogueira, G. Zeni, J. B. T. Rocha, Chem. Rev. 104 (2004) 6255; (e) G. Zeni, O. S.
D. Barros, A. V. Moro, A. L. Braga, C. Peppe, Chem. Commun. (2003) 1258; (e) J. P. Marino, H. N. Nguyen, J. Org. Chem. (67) 2002 6291; (f) A. V. Moro, C. W. Nogueira, N. B. V. Barbosa, P. H. Menezes, J. B. T. da Rocha, G. Zeni, J. Org. Chem. 70 (2005) 5257; (g) E. F. Lopes, L. C. Goncalves, J. C. G. Vinueza, R. G. Jacob, G. Perin, C. Santi, E. J. Lenardao, Tetrahedron Lett. 56 (2015) 6890; (h) B. Mohan, S. Hwang, H. Woo, K. H. Park, Tetrahedron 70 (2014) 2699; (i) A. Ogawa, H. Tanaka, H. Yokoyama, R. Obayashi, K. Yokoyama, N. Sonada, J. Org. Chem. 57 (1992) 111.

[2] (a) V. J. Blazis, K. J. Koeller, C. D. Spilling, J. Org. Chem. 60 (1995) 931; (b) A. Gautier, G. Garipova, O. Dubert, H. Oulyadi, S. R. Piettre, Tetrahedron Lett. 42 (2001) 5673; (c) T. F. Herpin, J. S. Houlton, W. B. Motherwell, B. P. Roberts, J. M. Weibel, Chem. Commun. (1996) 613; (d) C.-P. Chuang, S.-F. Wang, Synth. Commun. 25 (1995) 3459; (e) C. Lopin, G. Gouhier, A. Gautier, S. R. Piettre, J. Org. Chem. 68 (2003) 9916; (f) X. Huang, Q. Xu, C.-G. Liang, Q.-W. He, Synth. Commun. 32 (2002) 1243; (g) C. Lopin, A. Gautier, G. Gouhier, S. R. Piettre, Tetrahedron Lett. 41 (2000) 10195; (h) M. Marinozzi, M. C. Fulco, R. Rizzo, R. Pellicciari, Synlett (2004) 1027.

[3] (a) S. Mitra, S. Mukherjee, S. K. Sen, A. Hajra, Bioorg. Med. Chem. Lett. 24 (2014) 2198; (b) C. Lopin, G. Gouhier, A. Gautier, S. R. Piettre, J. Org. Chem. 68 (2003) 9916.

[4] L.-B. Han, N. Choi, M. Tanaka, J. Am. Chem. Soc. 118 (1996) 7000

[5] (a) Q. Xu, C.-G. Liang, X. Huang, Synth. Commun. 33 (2003) 2777; (b) J.-M. Chen, X. Huang, Synth. Commun. 34 (2004) 1745.

[6] Y.-X. Gao, G. Tang, Y. Cao, Y.-F. Zhao, Synthesis (2009) 1081.

[7] Y. Y. Li, S. H. Chen, L. Su, J. H. Li, X. H. Xu, Chin. J. Org. Chem. 33 (2013) 1999

[8] (a) L. Li, S. Shi, L. Song, L. Guo, Y. Wang, H. Ma, J. Hou, H. Wang, J. Organomet. Chem. 794 (2015) 231; (b) P. Bako, Z. Rapi, G. Keglevich, T. Szabo, P. L. Soti, T. Vigh, A. Grun, T. Holczbauer, Tetrahedron Lett. 52 (2011) 14736; (c) N. H. Khan, A. Sadhukhan, N. C. Maity, R. I. Kureshy, S. H. R. Abdi, S. Saravanan, H. C. Bajaj, Tetrahedron 67 (2011) 7073; (d) S. Nagayama, S. Kobayashi, J. Am. Chem. Soc. 122 (2000) 11531.

[9] (a) S. Kumar, N. K. Konduru, N. Verma, N. Ahmed, Synth. Commun. 45 (2015) 2555; (b) S. Kumar, N. Verma, N. Ahmed, RSC Adv. 5 (2015) 85128; (c) J. Shankar, G. Satish, B. S. P. A. Kumar, Y. V. D. Nageswar, Eur. J. Chem. 5 (2014) 668; (d) K. Ramesh, M. S. Narayana, K. Karnakar, Y. V. D. Nageswar, Tetrahedron Lett. 52 (2011) 4734; (e) R. Sridhar, B. Srinivas, V. P. Kumar, M. Narender, K. R. Rao, Adv. Synth. Catal. 349 (2007) 1873; (f) K. Surendra, N. S. Krishnaveni, R. Sridhar, K. R. Rao, J. Org. Chem. 71 (2006) 5819; (g) N. S. Krishnaveni, K. Surendra, K. R. Rao, Adv. Synth. Catal. 346 (2004) 346; (h) M. Komiyama, J. Am. Chem. Soc. 111 (1989) 3046.

[10] (a) K. Iwamoto, K. Araki, S. Shinkai, Tetrahedron 47 (1991) 4325; (b) W. Verboom, S. Datta, Z. Asfari, J. Org. Chem. 57 (1992) 5394; (c) V. Rawat, K. Press, I. Goldberg, A. Vigalok, Org. Biomol. Chem. 13 (2015) 11189; (d) S. Meninno, A. Parrella, G. Brancatelli, S. Geremia, C. Gaeta, C. Talotta, P. Neri, A. Lattanzi, Org. Lett. 17 (2015) 5100; (e) P. Sarkar, C. Mukhopadhyay, Green Chem. 17 (2015) 3452; (f) J. Gao, Z.-G. Ren, J.-P. Lang, J. Organomet. Chem. 792 (2015) 88.

[11] H. Taniguchi, Y. Otsuji, E. Nomura, Bull. Chem. Soc. Jpn. 68 (1995) 3563.

[12] R. Perrin, R. Lamartine, M. Perrin, Pure and Appl. Chem. 65 (1993) 1549 .

[13] (a) E. Nomura, H. Taniguchi, K. Kawaguchi, J. Org. Chem. 58 (1993) 4709; (b) S. Shinkai, S. Mori, H. Koreishi, J. Am. Chem. Soc. 108 (1986) 2409; (c) R. Cacciapaglia, A. Casnati, L. Mandolini, J. Am. Chem. Soc. (114) 1992 10956; (d) M. Ziołkowski, S. J. Grabowski, J. J. Leszczynski, Chem. Phy. 355 (2009) 169; (e) B. P. Hay, T. K. Firman, B. A. Moyer, J. Am. Chem. Soc. 127 (2005)1810.

[14] Ouyang,Y. J.; Qiu, R. H.; Chen, S. H.; Chen, J. Y.; Xu, X. H. Chin. J. Org. Chem. 2015, 35, 2636-2641.

[15] V. Percec, T. K. Bera, B. B. De, J. Org. Chem. 66 (2001) 2104.

[16] J. S. Kim, O. J. Shon, J. W. Ko, M. H. Cho, I. Y. Yu, J. Vicens, J. Org. Chem. 65 (2000) 2386.

[17] W. L. Dong, G. Y. Huang, Z. M. Li, W. G. Zhao, Phosphorus, Sulfur, and Silicon and the other related Elements 184 (2009) 2058.

[18] (a) Y. He, D. Huang, M. Lu, Phosphorus, Sulfur, and Silicon and the other related Elements 187 (2012) 1118; (b) Y. J. Ouyang, Y. Y. Li, N. B. Li, X. H. Xu, Chin. Chem. Lett. 24 (2013) 1103 